\title{
RA?EGA
}

O ESPACYO GEOGRÁFICO EM ANÁLISE

\section{PROPRIEDADES FÍSICAS E HIDROLÓGICAS DOS SOLOS E OS ESCORREGAMENTOS RASOS NA SERRA DO MAR PAULISTA}

\author{
PHYSICAL AND HYDROLOGIAL SOIL PROPERTIES AND THE \\ SHALLOW LANDSLIDES IN THE SERRA DO MAR PAULISTA
}

\author{
Bianca Carvalho Vieira \\ Departamento de Geografia \\ Universidade de São Paulo (USP) \\ São Paulo, SP \\ e-mail:biancacv@usp.br \\ Fabiana Souza Ferreira \\ Centro Universitário Fundação Santo André \\ São Paulo, SP \\ e-mail:fa_s_f@yahoo.com.br \\ Maria Carolina Villaça Gomes \\ Programa de Pós-Graduação em Geografia Física \\ Universidade de São Paulo (USP) \\ São Paulo, SP \\ e-mail: mariacarolinavg@usp.br
}

\section{Recebido em: 07/04/2015}

Aceito em: 02/09/2015

\section{Resumo}

O objetivo deste trabalho foi determinar a influência de algumas propriedades físicas e hidrológicas do solo na deflagração dos escorregamentos translacionais rasos na Serra do Mar (SP). Foram selecionadas três cicatrizes de escorregamentos rasos deflagrados durante o evento de 1967, nas quais foram coletadas amostras em três pontos (lateral, superior e interior) e em diferentes profundidades, sendo analisadas as seguintes propriedades: granulometria, porosidade, coesão, ângulo de atrito interno e condutividade hidráulica saturada. Os resultados mostraram altos valores de areia e baixos percentuais de argila, com reduzidos índices de atividade. Os valores de coesão variaram entre $0 \mathrm{kPa}$ e $11 \mathrm{kPa}$ e o ângulo de atrito interno entre $25,8^{\circ}$ e $36,8^{\circ}$. A condutividade hidráulica saturada apresentou pequena variabilidade, mas significativas descontinuidades entre 1 e 2,5 m de profundidade. Acredita-se que este estudo possa fornecer informações para definição de locais e profundidades para o monitoramento hidrológico em tempo real, contribuindo para o entendimento e para a previsão dos escorregamentos rasos na Serra do Mar.

Palavras-chave: Coesão, ângulo de atrito interno, condutividade hidráulica saturada. 


\title{
PROPRIEDADES FÍSICAS E HIDROLÓGICAS DOS SOLOS E OS ESCORREGAMENTOS RASOS NA SERRA DO MAR PAULISTA
}

\begin{abstract}
The aim of this paper was to determine the influence of some physical and hydrological soil properties on trigger shallow landslides in the Serra do Mar (SP). We selected three landslides scars occurred in 1967 event, where were collected samples at the sites (lateral, top and in the center) and in different depths, being analyzed the following properties: texture, porosity, cohesion, angle of internal friction and saturated hydraulic conductivity. The results shows high values of sand content and low values of clay, which had reduced activity index. The cohesion values ranged between $0 \mathrm{kPa}$ to $11 \mathrm{kPa}$, and the angle of internal friction between $25.8^{\circ}$ and $36.8^{\circ}$. Saturated hydraulic conductivity presented low variability, but significant discontinuities between 1 and $2.5 \mathrm{~m}$. We believe this study can provide data to define sites and depths for real-time hydrological monitoring and consequently contribute to understand and predict shallow landslides in the Serra do Mar.
\end{abstract}

Keywords: Cohesion, angle of internal friction, saturated hydraulic conductivity.

\section{INTRODUÇÃO}

No Brasil, dentre os principais desastres naturais, os movimentos de massa têm sido responsáveis por grande parte do número de vítimas fatais e prejuízos materiais. A Serra do Mar, compartimento geológico-geomorfológico que se estende por mais $1.500 \mathrm{Km}$ no litoral sul/sudeste, é uma das áreas mais afetadas por estes processos. Nesta área, os estudos sistemáticos sobre estes processos iniciaram ainda na década de 1920. Em 1929, após fortes chuvas, deslizamentos interromperam o tráfego da estrada de ferro Santos-Jundiaí por 15 dias e seus detritos atingiram, inclusive, as instalações subterrâneas da estrada de ferro (VARGAS, 1999). Porém, os mais importantes já registrados ocorreram nos morros de Santos em 1928 e posteriormente em 1956. Nesse último ano a precipitação pluviométrica atingiu o valor de $373 \mathrm{~mm}$ em 24horas, deflagrando quase 60 escorregamentos simultaneamente (IPT, 1986).

Nos dias 17 e 18 de março de 1967, no município de Caraguatatuba (SP), foram registrados, respectivamente, $260 \mathrm{~mm}$ e $324,8 \mathrm{~mm}$, ou seja, cerca de $35 \%$ da pluviosidade anual daquela região (IPT, 1988). Como consequência, ocorreram escorregamentos generalizados que, após serem canalizados pela rede de drenagem (Figura 1), se transformaram rapidamente em corridas de detritos com grande mobilização de material, ocorrendo num raio variando de 7 a 15 quilômetros 


\section{PROPRIEDADES FÍSICAS E HIDROLÓGICAS DOS SOLOS E OS ESCORREGAMENTOS RASOS NA SERRA DO MAR PAULISTA}

de extensão (GRAMANI, 2001). Neste verão, o município registrou 120 mortes e 400 casas destruídas e, até hoje, são visíveis as cicatrizes destes processos.
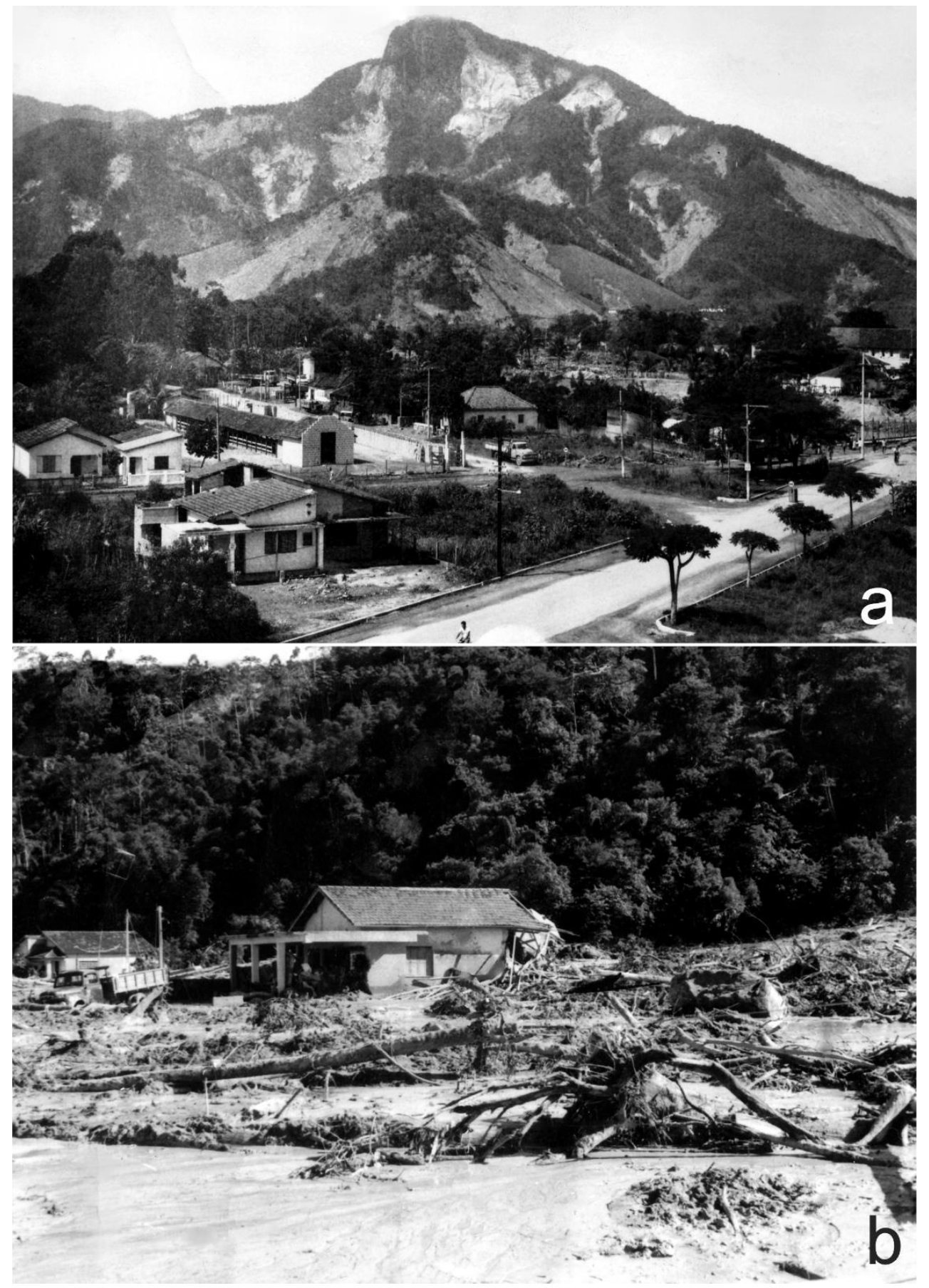

Figura 1: Escorregamentos generalizados no munícipio de Caraguatatuba em março de 1967. (a) observa-se as cicatrizes nas encostas em grande magnitude e (b) parte das corridas de detritos que atingiram a planície e mobilizaram um grande volume de árvores (Fonte: Arquivo Municipal de Caraguatatuba). 
Nos dias 11 e 12 de janeiro 2011, cerca de oito municípios da região serrana do Rio de Janeiro foram afetados por 3.562 escorregamentos (AVELAR et al., 2011), sobretudo no contato solo/rocha e colúvio/rocha, e corridas de detritos que atingiram mais de $20 \mathrm{~km}$ de distância e foram, em grande parte, responsáveis por mais de 1.500 mortes e cerca de 20.000 desabrigados. A deflagração foi ocasionada por uma associação de fortes chuvas antecedentes entre os meses de outubro e dezembro, culminando nestes dois dias em cerca de $300 \mathrm{~mm} / 48 \mathrm{~h}$, segundo os autores.

Estes números revelam a magnitude destes processos, que, mesmo sendo responsáveis por grandes danos, ainda pouco se conhece sobre as condições que levam à sua deflagração, sobretudo em relação à dinâmica hidrológica, que pode fornecer informações determinantes para um melhor entendimento dos mecanismos de desestabilização das encostas. Conforme apontam van Asch et al. (1999), as propriedades físicas e hidrológicas dos solos, nas áreas potencialmente instáveis a movimentos de massa, podem determinar a frequência e a tipologia destes processos, sob as mesmas condições meteorológicas. Dentre estas propriedades, destacam-se, por exemplo, a granulometria, a coesão e a condutividade hidráulica saturada $\left(\mathrm{K}_{\text {sat }}\right)$. Esta última pode influenciar direta ou indiretamente os mecanismos de ruptura e a consequente deflagração dos escorregamentos rasos (SIDLE et al., 1985; WOLLE e HACHICH, 1989; SELBY, 1993; TERLIEN, 1997).

$\mathrm{Na}$ literatura, dois mecanismos de ruptura são amplamente relacionados à variação da $\mathrm{K}_{\text {sat. }}$ Para alguns autores, as rupturas podem ocorrer quando, no manto de alteração, há presença de materiais mais permeáveis em contato direto com materiais menos permeáveis, favorecendo o desenvolvimento rápido de poropressões positivas. Um outro tipo de mecanismo de ruptura está associado à perda de sucção e à redução da coesão aparente devido ao aumento continuo da $\mathrm{K}_{\text {sat }}$ em profundidade. Este aumento pode estar condicionado, por exemplo, à presença de sistemas de fraturamento no manto de alteração, que permitem a drenagem da água subsuperficial e dificultam o processo de saturação total do solo (CAMPOS et al., 1992; BRUGGER et al., 1997; LACERDA et al., 1997; TERLIEN, 1997; VIEIRA E FERNANDES, 2004; GERSCOVICH et al., 2006; entre outros). 
Assim, conforme exposto, o levantamento in situ das propriedades físicas e hidrológicas, em diferentes locais na paisagem e em profundidades, é fundamental para o entendimento da dinâmica hidrológica durante eventos intensos de chuva que podem gerar condições de instabilidade das encostas. Desta forma, o objetivo deste trabalho foi determinar a influência de algumas propriedades físicas e hidrológicas do solo na deflagração dos escorregamentos translacionais rasos na Serra do Mar (SP).

\section{2. ÁREA DE ESTUDO - BACIA DO RIO GUAXINDUBA}

O município de Caraguatatuba (SP) tem cerca de $60 \%$ da sua área constituída por serras e morros e outros e $40 \%$ por planícies litorâneas. A bacia do rio Guaxinduba (Figura 2) compreende uma área de $24 \mathrm{Km}^{2}$, constituída por planalto e escarpas da Serra do Mar, sendo esta última mais expressiva da área de estudo, onde se encontram encostas de forte declividade (cerca de 40\%) e o predomínio de gnaisses, metassedimentos e feições estruturais (falhas, fraturas e foliações) bem delimitadas e com orientações, em sua maior parte, para NE-SW (ALMEIDA, 1964; HASSUI et al., 1994).

Como resultado dos tipos de rochas que compõem a bacia do Guaxinduba, segundo Cruz (1974), os solos configuram-se com uma camada superficial de 1 a 2 $\mathrm{m}$, a camada logo abaixo, com precipitação do ferro, numa espessura de 2 a $5 \mathrm{~m}$ de profundidade que corresponderia ao horizonte $\mathrm{B}$ que pode aflorar por meio da ação de processos erosivos, por exemplo. $O$ horizonte $C$ apresenta grandes espessuras, conservando a mesma estrutura da rocha mãe, sem alteração de volume; mas também se torna pouco consistente, transformada quimicamente pela caolinização dos feldspatos, cada vez mais acessível à penetração da água. Abaixo ocorre a camada pouco alterada, com matacões de rocha sã com maior frequência, de acordo com as linhas estruturais (De PLOEY E CRUZ, 1979).

A vegetação pertence ao domínio morfoclimático de Mata Atlântica, subdividida em duas formações florestais principais: mata de encosta, que possui árvores altas com dossel descontinuo; e mata de altitude, que se caracteriza por ocorrer acima de $1100 \mathrm{~m}$. Além da mata, verifica-se alta taxa de ocorrência de capoeira. Como consequências dos escorregamentos de 1967 uma grande parte da 


\section{PROPRIEDADES FÍSICAS E HIDROLÓGICAS DOS SOLOS E OS ESCORREGAMENTOS RASOS NA SERRA DO MAR PAULISTA}

vegetação foi remobilizada e as cicatrizes foram recobertas por vegetação rasteira do gênero Gleychenia, que se adapta em solos rasos.

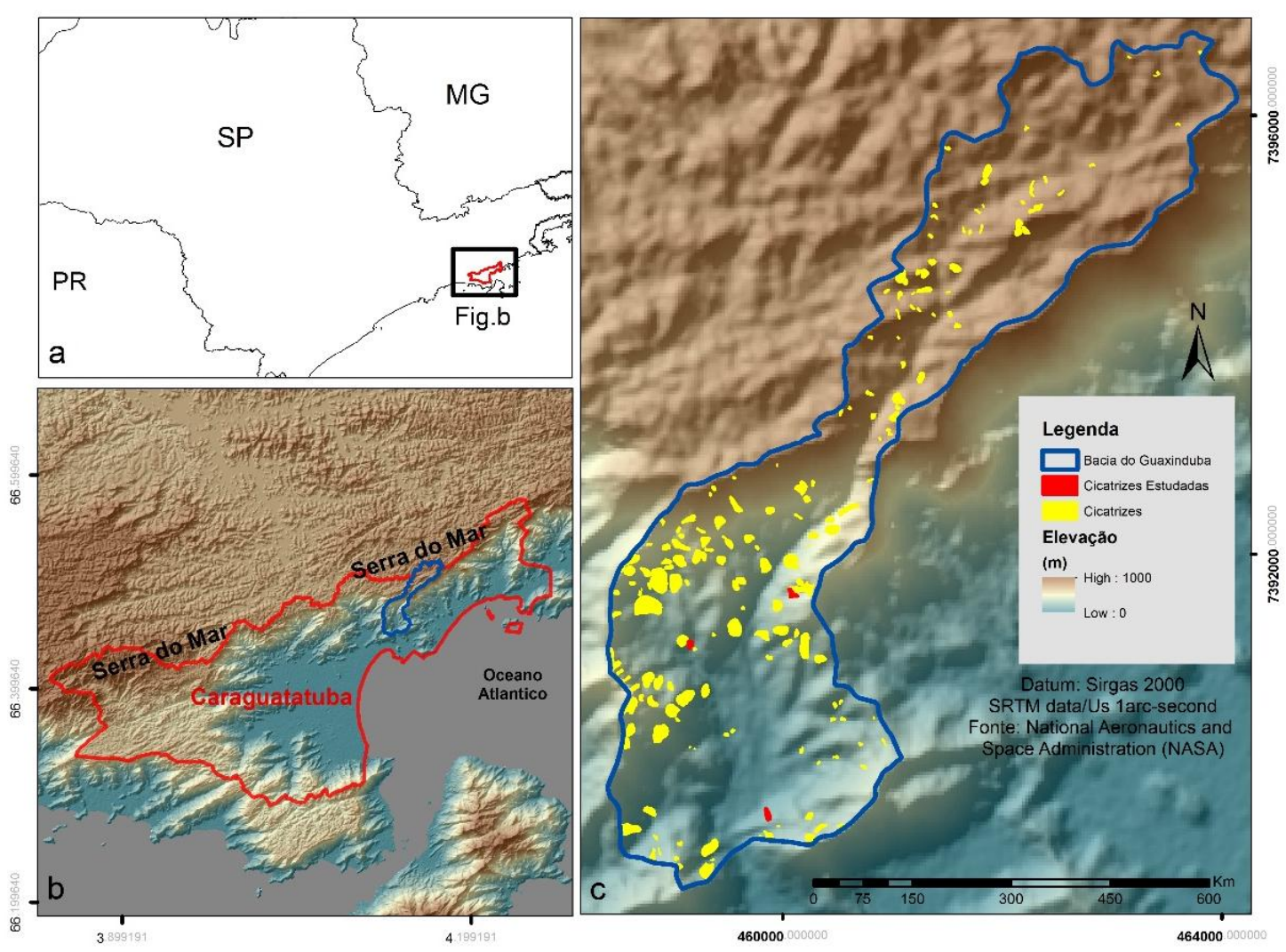

Figura 2: (a) Município de Caraguatatuba no estado de São Paulo, (b) localização da bacia do rio Guaxinduba e (c) bacia do rio Guaxinduba com as cicatrizes de 1967 (em amarelo) com destaque para aquelas que foram estudadas in situ (em vermelho).

\section{MATERIAIS E MÉTODOS}

Para o levantamento de campo e análise das propriedades dos solos, foram selecionadas três cicatrizes de escorregamentos rasos deflagrados em 1967 (C1, C2 e C3) considerando seus limites bem preservados e o acesso para coleta das amostras (Figura 2). Em cada uma das cicatrizes foram abertos três perfis, até $2 \mathrm{~m}$ de profundidade, no topo (P1), no centro (P2) e na lateral (P3) (Figura 3). A definição do local dos perfis baseou-se na premissa de que o material mobilizado se assemelha ao do topo e da lateral da cicatriz, ou seja, ao sobrepor os perfis do topo (ou lateral) e do centro têm-se o manto de alteração completo anterior à ruptura. 

NA SERRA DO MAR PAULISTA
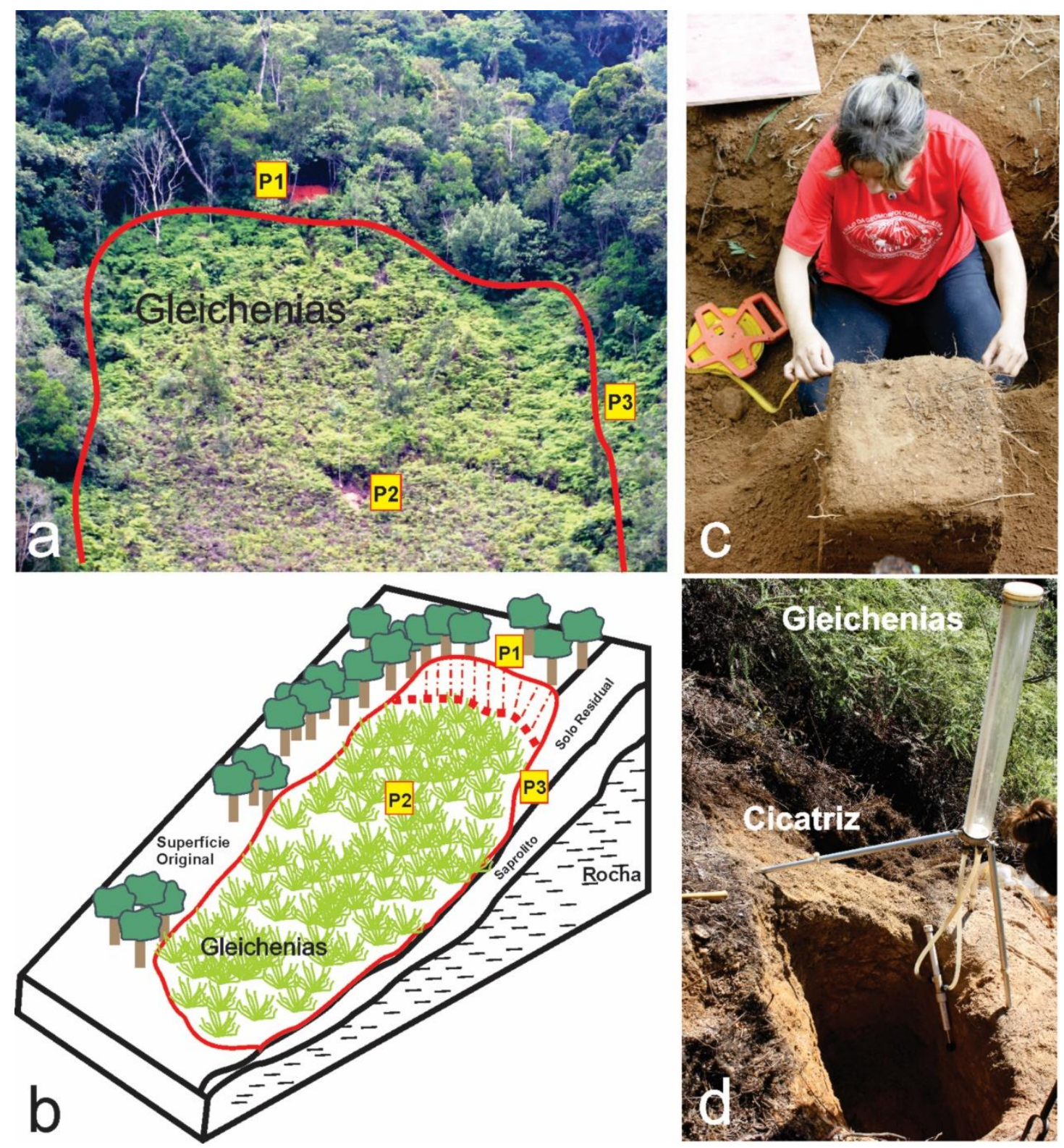

Figura 3: (a) Localização dos perfis ( $\mathrm{P} 1, \mathrm{P} 2$ e $\mathrm{P} 3$ ) em relação aos limites da cicatriz do escorregamento (limite em vermelho). (b) Desenho esquemático dos perfis em relação à cicatriz, (c) exemplo da coleta de uma amostra deformada para ensaio de coesão e (d) Permeâmetro de Guelph em campo para coleta da condutividade hidráulica saturada. Fonte: Autores.

Em cada um desses perfis foram retiradas amostras deformadas e indeformadas em diferentes profundidades a medida que foram observadas alterações texturais (tátil-visuais) do solo, na tentativa de melhor caracterizar as alterações ao longo do perfil pedológico.

Foram coletadas 37 amostras deformadas para a realização de ensaios de granulometria e 6 blocos indeformados, de dimensões $30 \times 30 \times 30 \mathrm{~cm}$, para coesão (c') e ângulo de atrito $\left(\varphi^{\prime}\right)$, nas seguintes profundidades: C1 $(0,40 \mathrm{~m}$ e $1,75 \mathrm{~m}), \mathrm{C} 2$ 
$(0,60 \mathrm{~m}$ e $2,00 \mathrm{~m})$ e $\mathrm{C} 3(0,45 \mathrm{~m}$ e $2,00 \mathrm{~m})$. Ressalta-se que a resistência ao cisalhamento dos solos foi definida pelas envoltórias de ruptura obtidas nos ensaios de cisalhamento direto inundado. Os resultados obtidos a partir desses ensaios foram analisados e comparados com algumas propriedades físicas, visando identificar as variáveis responsáveis pelo controle dos parâmetros de resistência ao cisalhamento (c', $\left.\varphi^{\prime}\right)$.

No total, para ensaios de porosidade (total, macro e micro) foram coletadas 24 amostras de prova em anéis cilíndricos biselados de PVC com $5 \mathrm{~cm}$ de altura e $5 \mathrm{~cm}$ de diâmetro interno até as seguintes profundidades: C1 $(1,45 \mathrm{~m}), \mathrm{C} 2(1,00 \mathrm{~m}) \mathrm{e}$ C3 $(1,47 \mathrm{~m})$ e sendo o utilizado para este ensaio o método da mesa de tensão conforme descrito por Kiehl (1979). É importante destacar que devido à grande resistência do material amostrado e à abertura das trincheiras (perfis), a profundidade máxima alcançada na coleta de amostras deformadas para granulometria $(\sim 5 \mathrm{~m})$ foi bem maior daquela alcançada para as amostras indeformadas $(\sim 1,47 \mathrm{~m})$.

O levantamento da condutividade hidráulica saturada $\left(\mathrm{K}_{\text {sat }}\right)$ foi realizado utilizando o Permeâmetro de Guelph, nas profundidades $0,25,0,50,1,00,1,50,2,00$ e 2,50m, definidas a partir da caracterização morfológica tátil-visual dos solos de todos os perfis, e com a sobreposição, os perfis completos perfizeram $5 \mathrm{~m}$ de profundidade.

O Permeâmetro de Guelph foi desenvolvido por Reynolds et al. (1983) tendo como principais vantagens: ser leve, operável por uma pessoa, por causar mínimas perturbações no solo e, em relação aos outros métodos, utiliza pouca água, com baixo custo e com demanda menor de tempo para a obtenção dos resultados finais (REYNOLDS e ELRICK, 1985).

\section{RESULTADOS E DISCUSSÃO}

\subsection{Granulometria e Porosidade}

De maneira geral as amostras apresentaram valores altos da fração de areia sobretudo nas cicatrizes 1 e 2, com valores variando entre $60 \%$ e $70 \%$, porém com percentuais bem mais altos (>80\%) a partir de $2,55 \mathrm{~m}$ de profundidade em C1 (Figura $4)$. 


\section{PROPRIEDADES FÍSICAS E HIDROLÓGICAS DOS SOLOS E OS ESCORREGAMENTOS RASOS NA SERRA DO MAR PAULISTA}
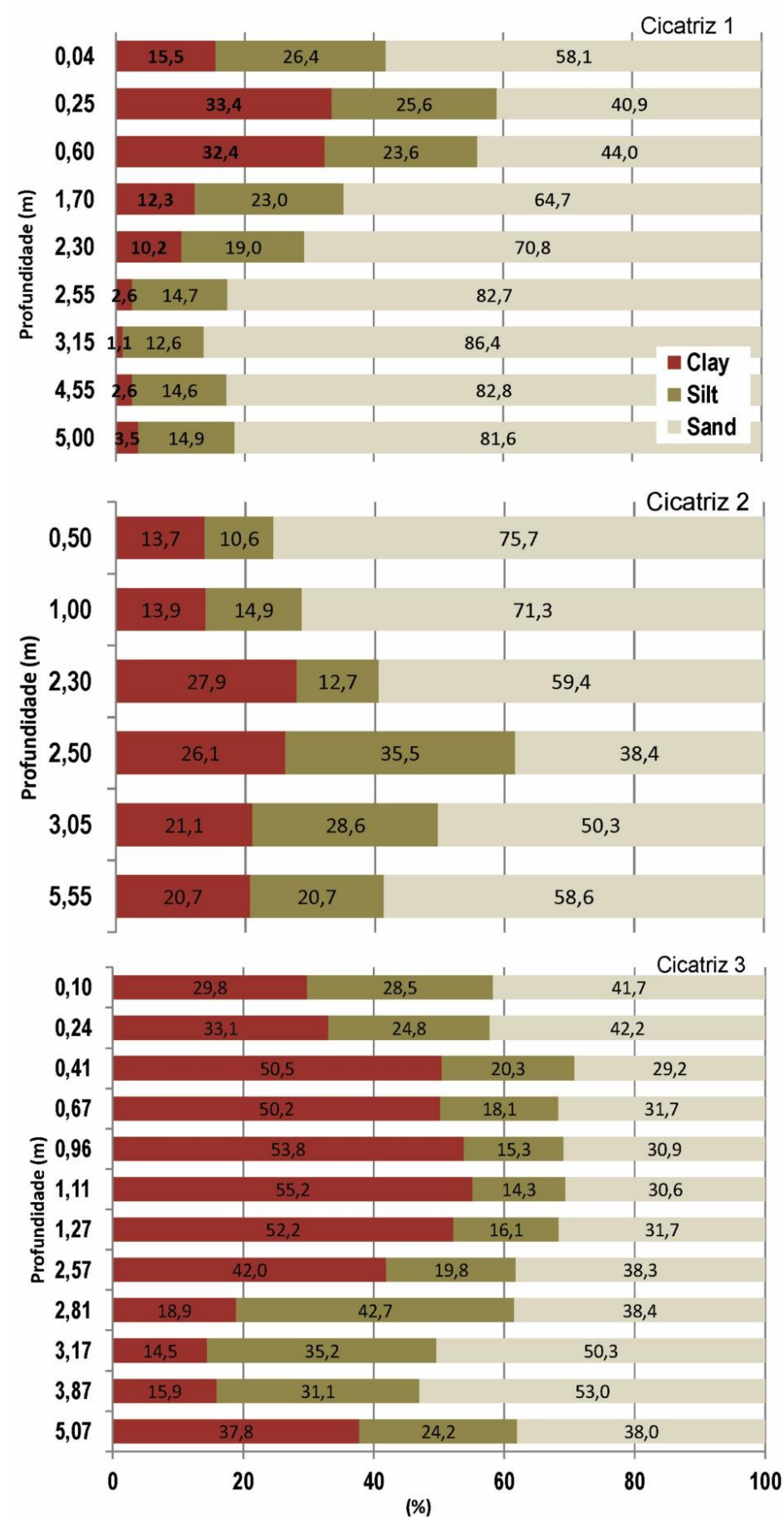

Figura 4: Valores percentuais de argila, silte e areia em diferentes profundidades nas Cicatrizes 1, 2 e 3.

Em geral os horizontes mais superficiais ( 1m) apresentaram um percentual maior da fração argila, que alcançou quase $55 \%$ em $1 \mathrm{~m}$ de profundidade na Cicatriz 3. Valores similares foram encontrados por Mendes (2008) em perfis de solos na 
região de Ubatuba suscetíveis a escorregamentos que, de modo geral, apresentou uma redução do teor de argila com aumento da profundidade.

Conforme a Figura 4, na $\mathrm{C} 1$ foi observado um alto percentual de argila até a profundidade de $0,60 \mathrm{~m}$ e a partir daí um aumento expressivo da fração areia, que alcançou cerca de $87 \%$ em $3 \mathrm{~m}$ de profundidade. C2 e C3 apresentaram altos valores médios de argila em todo o perfil, com destaque para o maior teor de argila, em média, em C3 com cerca de 38\% e valores acima de 50\% entre $0,41 \mathrm{~m} \mathrm{e} 1,27 \mathrm{~m}$.

Em relação à porosidade total foram registrados valores médios entorno de $65 \%$ em todas as cicatrizes (Figura 5), com predomínio de microporos. Apenas C3 apresentou um claro aumento da porosidade total com a profundidade, conforme também observado por Mendes (2008) que verificou um maior volume de poros nas maiores profundidades. Em relação aos microporos, o autor identificou tanto o seu predomínio nos horizontes mais superficiais quanto perfis com uma distribuição mais uniforme entre macro e microporos. Destaca-se aqui, segundo o autor, que um dos perfis apresentou significativo volume de microporos tanto nos horizontes superficiais quanto no saprolito.

Considerando a granulometria até a profundidade de $2 \mathrm{~m}$ (máxima alcançada para porosidade), observou-se uma relação direta entre o aumento do teor de argila e a microporosidade em todas as cicatrizes.

\subsection{Condutividade Hidráulica Saturada $\left(\mathrm{K}_{\text {sat }}\right)$}

Foram obtidos 41 valores de $\mathrm{K}_{\text {sat }}$, que variaram entre três ordens de grandeza $\left(10^{-6} \mathrm{~m} / \mathrm{s}\right.$ a $\left.10^{-4} \mathrm{~m} / \mathrm{s}\right)$, embora mais de $80 \%$ tenham se concentrado entre $10^{-6} \mathrm{~m} / \mathrm{s}$ a $10^{-}$ ${ }^{5} \mathrm{~m} / \mathrm{s}$. A K $\mathrm{K}_{\text {sat }}$, em profundidade, apresentou variações distintas, com a tendência de aumento em profundidade e a presença de descontinuidades hidráulicas significativas (Figura 6).

$\mathrm{Na} \mathrm{C} 1$, os valores variaram entre $10^{-6} \mathrm{~m} / \mathrm{s}$ a $10^{-4} \mathrm{~m} / \mathrm{s}$ até $2,5 \mathrm{~m}$, foram obtidos valores considerados altos $\left(10^{-5} \mathrm{~m} / \mathrm{s}\right.$ a $\left.10^{-4} \mathrm{~m} / \mathrm{s}\right)$ e com pequena amplitude. Esses valores podem ser atribuídos aos altos percentuais de porosidade, mas sobretudo aos elevados teores de areia, bem como à existência de blocos centimétricos imersos na matriz coluvionar. Entre 2,5m e 2,75m, houve uma brusca redução de cerca de 100 vezes $\left(10^{-4} \mathrm{~m} / \mathrm{s}\right.$ para $\left.10^{-6} \mathrm{~m} / \mathrm{s}\right)$, já no saprolito pouco alterado. Fato este 


\section{PROPRIEDADES FÍSICAS E HIDROLÓGICAS DOS SOLOS E OS ESCORREGAMENTOS RASOS NA SERRA DO MAR PAULISTA}

que pode estar associado à queda brusca do teor de argila a partir desta profundidade, que alcançou valores inferiores a $2 \%$ (Figura 6).

$\mathrm{Na} C 2$ os valores variaram entre $10^{-6} \mathrm{~m} / \mathrm{s}$ a $10^{-5} \mathrm{~m} / \mathrm{s}$, com descontinuidades hidráulicas menos acentuadas. Até $2,5 \mathrm{~m}$ (no solo residual) houve uma tendência de aumento com a profundidade $\left(10^{-6} \mathrm{~m} / \mathrm{s}\right.$ aos, $0,25 \mathrm{~m}$, para $10^{-5} \mathrm{~m} / \mathrm{s}$, aos $\left.2,5 \mathrm{~m}\right)$, e posterior redução até $3,5 \mathrm{~m}$ no saprolito. Este aumento seguido por uma leve redução pode ser explicado pela variação do conteúdo de areia e da macroporosidade, com uma redução até $2,5 \mathrm{~m}$ e posterior aumento até $3,5 \mathrm{~m}$.

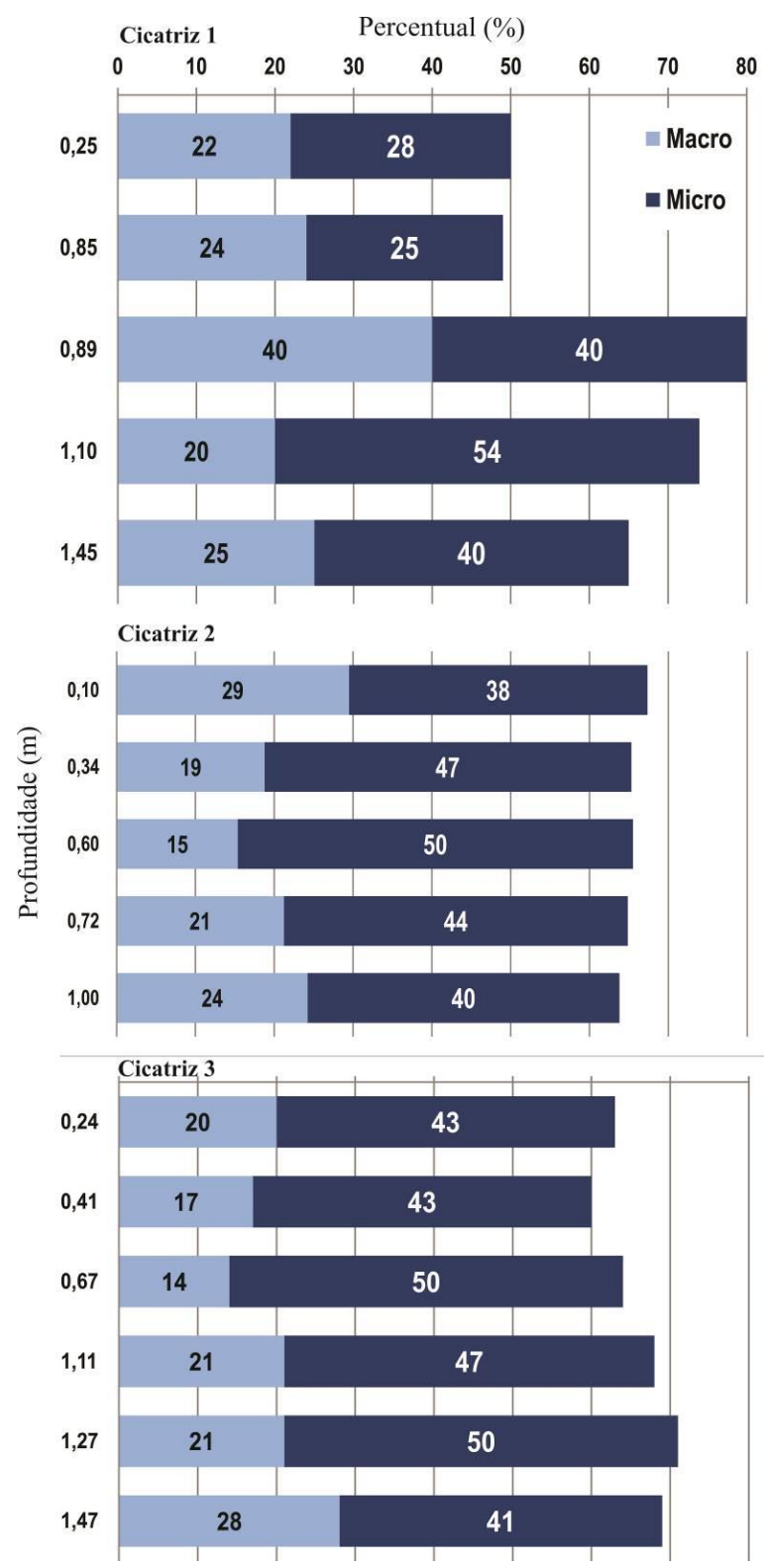

Figura 5: Valores percentuais de macro e microporos em diferentes profundidades nas Cicatrizes 1, 2 e 3 . 


\section{PROPRIEDADES FÍSICAS E HIDROLÓGICAS DOS SOLOS E OS ESCORREGAMENTOS RASOS NA SERRA DO MAR PAULISTA}
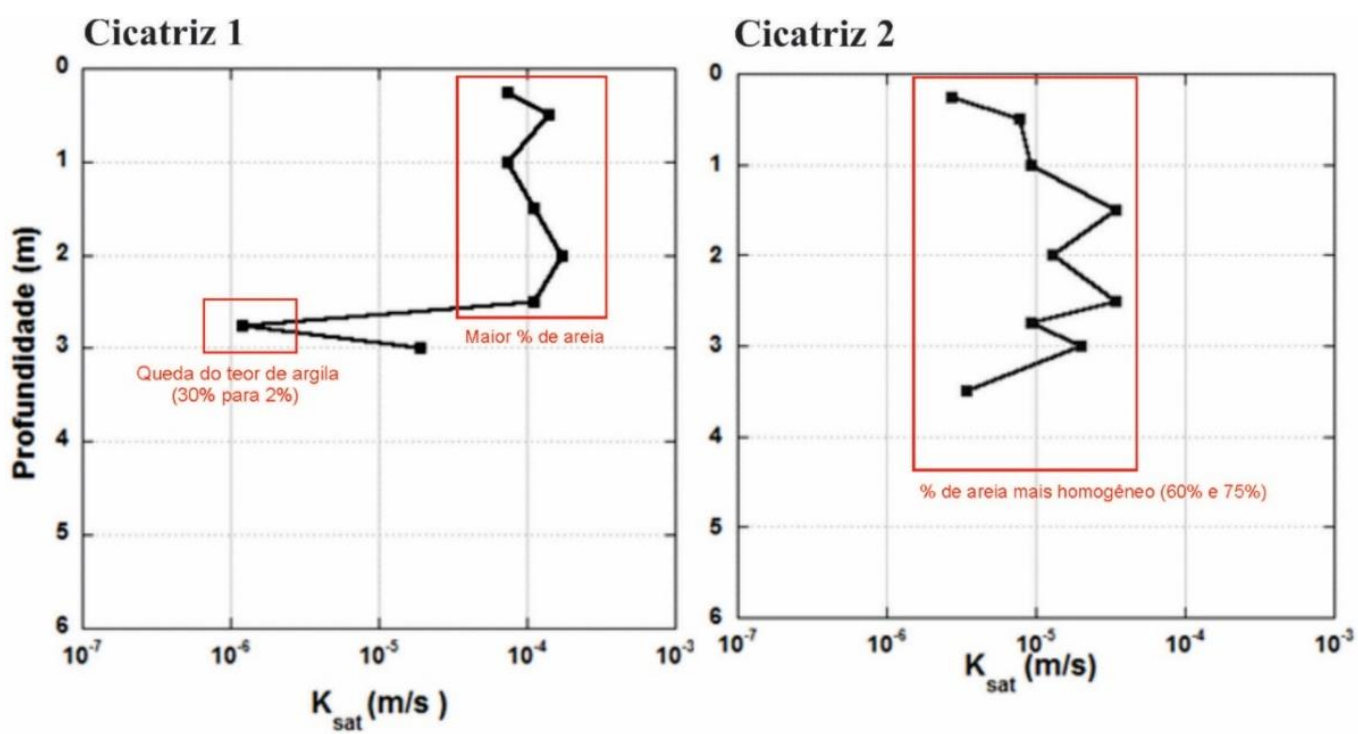

Cicatriz 3

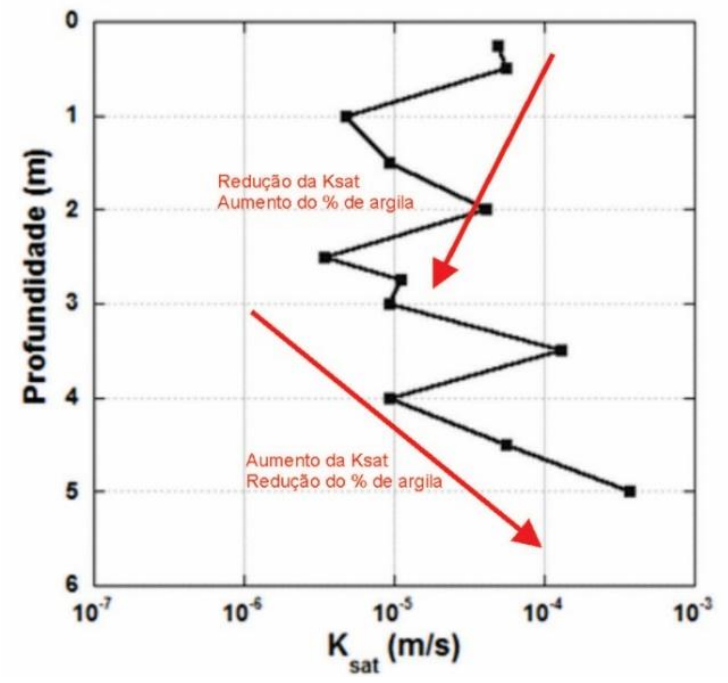

Figura 6: Variação da $\mathrm{K}_{\text {sat }}$ em profundidade nas cicatrizes 1, 2 e 3. Na cicatriz 1 observa-se um elevado percentual da $\mathrm{K}_{\text {sat }}$ e do teor de areia. Na cicatriz 2 um percentual de areia mais homogêneo e na cicatriz 3 um aumento da $\mathrm{K}_{\text {sat }}$ até cerca de $2,5 \mathrm{~m}$ com posterior aumento acompanhado pela redução do teor de argila.

Na C3 foi observado uma variação da $\mathrm{K}_{\text {sat }}$ diretamente relacionada aos teores de argila (Figura 6). Até $3 \mathrm{~m}$ uma tendência a redução $\mathrm{K}_{\text {sat }}$ com aumento do teor de argila (média de $45 \%$ ) e posterior aumento da $\mathrm{K}_{\text {sat }}$ paralelamente à redução do teor de argila e, consequente, aumento do percentual de areia, que alcançou cerca de $70 \%$ em $5 \mathrm{~m}$ de profundidade.

As descontinuidades identificadas nas cicatrizes podem desempenhar um papel muito importante na dinâmica hidrológica dos solos e, consequentemente, na estabilidade das encostas. Em locais onde há alterações abruptas na $\mathrm{K}_{\text {sat, }} \mathrm{O}$ 
aumento do grau de saturação pode levar à formação de um nível freático suspenso e ao desenvolvimento rápido de poro-pressões positivas, que reduzem a tensão efetiva do material. Destaca-se também a importância da profundidade onde tais descontinuidades foram observadas, sendo que das quatro identificadas, três se encontram entre $1 \mathrm{~m}$ e $2,5 \mathrm{~m}$. Tal comportamento já foi identificado em outros trabalhos como sendo uma profundidade crítica para as encostas da Serra do Mar como por exemplo por Wolle e Hachich (1989), Campos et al. (1992) e Mendes (2008).

\subsection{Coesão e Ângulo de Atrito}

Conforme a Tabela 1 , os valores de coesão variaram entre $0 \mathrm{kPa}$ e $11 \mathrm{kPa}$ e o ângulo de atrito interno entre $25,8^{\circ}$ e $36,8^{\circ}$, sendo ambos mais elevados nas maiores profundidades, exceto na cicatriz 3 em que foi verificado valor de $0 \mathrm{kPa}$ em ambas as profundidades. Em C1 e C2 as camadas superficiais $(0,40 \mathrm{~m}$ e $0,60 \mathrm{~m})$ apresentaram ângulos de atrito menores $\left(29,1^{\circ}\right.$ e $\left.25,8^{\circ}\right)$ em detrimento das camadas sobrejacentes, $1,75 \mathrm{~m}$ e $2 \mathrm{~m}$ (31,9o e 36,8). Contrariamente, em C3, sua camada superficial $(0,45 \mathrm{~m})$ apresentou ângulo de atrito maior $\left(36,7^{\circ}\right)$ em distintamente da camada subjacente, $2 \mathrm{~m}\left(33,5^{\circ}\right)$.

Tabela 1: Valores de coesão ( $\left.C^{\prime}\right)$ e ângulo de atrito $(\Phi)$ para as cicatrizes $C 1, C 2$ e C3 em distintas profundidades $(\mathrm{m})$.

\begin{tabular}{|c|c|c|}
\hline Cicatriz (Prof.) & $\mathbf{C '}^{\prime}(\mathbf{k P a})$ & $\mathbf{\Phi}\left(\mathbf{(}^{\mathbf{}}\right)$ \\
\hline C1 $(0,60)$ & 2,16 & 25,8 \\
\hline C1 $(2)$ & 11 & 36,8 \\
\hline C2 $(0,40)$ & 0 & 29,1 \\
\hline C2 1,75 & 1,2 & 31,9 \\
\hline C3 $(0,45)$ & 0 & 36,7 \\
\hline C3 $(2)$ & 0 & 33,5 \\
\hline
\end{tabular}

Collins e Znidarcic (1997), investigando as características dos solos e da morfologia relacionadas a escorregamentos e a corridas de detritos em Santa Cruz (Canadá), apontaram que uma das causas internas dos escorregamentos é a alteração do equilíbrio entre as tensões no interior da massa. Muitas vezes as 


\section{PROPRIEDADES FÍSICAS E HIDROLÓGICAS DOS SOLOS E OS ESCORREGAMENTOS RASOS NA SERRA DO MAR PAULISTA}

frentes de umedecimento localizam-se no contato entre rocha sã ou rocha parcialmente decomposta, assim, esses materiais que contêm mais areia e menor quantidade de argila, atingem mais rapidamente o limite de liquidez. Este, por sua vez, está preso à plasticidade das argilas, que como visto nesse trabalho, são argilas inativas, dificultando assim a capacidade de retenção de água.

De acordo com a Tabela 2, valores de coesão bem próximos foram encontrados nos trabalhos de Wolle e Carvalho (1994), Amaral Junior (2007) e Silva (2006) em diferentes partes da Serra do Mar. Wolle e Carvalho (1994), por exemplo, por meio de ensaios in situ e de laboratório em duas áreas pilotos na região de Cubatão (SP), encontraram características muito próximas a essa pesquisa, solos areno-argilosos e arenosos, com camadas superficiais apresentando ângulo de atrito menor $\left(34^{\circ}\right)$ em detrimento da camada sobrejacente $\left(39^{\circ}\right)$ e valores para coesão entre 1 e $4 \mathrm{kPa}$.

Tabela 2: Valores de coesão (C'), em kPa; ângulo de atrito $(\Phi)$, em graus obtidos em diferentes profundidades (metros) na Serra do Mar.

\begin{tabular}{|c|c|c|c|c|c|}
\hline Autor & Prof. & C' $^{\prime}$ & $\phi$ & Litologia & Local \\
\hline $\begin{array}{c}\text { Wolle e } \\
\text { Carvalho (1994) }\end{array}$ & $1-2$ & $1-4$ & $34-39$ & Migmatito & $\begin{array}{c}\text { Cubatão } \\
\text { (SP) }\end{array}$ \\
\hline Autores & $0,45-2$ & $0-11$ & $25-37$ & Gnaisse & $\begin{array}{c}\text { Caraguatatuba } \\
\text { (SP) }\end{array}$ \\
\hline $\begin{array}{c}\text { Amaral Junior } \\
\text { (2007) }\end{array}$ & $1.5-5$ & 8 & 32 & $\begin{array}{c}\text { Biotita } \\
\text { Gnaisse }\end{array}$ & $\begin{array}{c}\text { Costa Verde } \\
\text { (RJ) }\end{array}$ \\
\hline Silva (2006) & 1 & $0-9$ & $38-39$ & - & Petrópolis (RJ) \\
\hline
\end{tabular}

Amaral Junior (2007) observou as envoltórias de resistência ao cisalhamento saturado apresentaram uma inclinação constante ao longo de toda a faixa de tensões normais, obtendo um valor de coesão igual a $8 \mathrm{kPa}$ e ângulo de atrito igual a $32^{\circ}$. O autor teve como proposta o mapeamento geotécnico aplicado à análise dos diferentes tipos de ocorrência dos movimentos de massa gravitacionais na região de Costa Verde (RJ). Segundo o autor, a agregação destes solos, especialmente nas camadas superficiais, contendo $70 \%$ de areia e 10\% de argila não apresentaram resistência para produzir mudanças significativas na inclinação e no intercepto da envoltória, portanto, pôde-se afirmar que mesmo para tensões normais baixas empregadas (14 $\mathrm{kPa}$ ) seriam suficientes para romper os agregados deste solo, de maneira que a envoltória foi definida por suas características granulométricas, assim 
como os valores encontrados na presente pesquisa, caracterizados por $80 \%$ de areia e $10 \%$ de argila.

\section{CONSIDERAÇÕES FINAIS}

Com o objetivo de investigar e analisar a influência das propriedades físicas e hidrológicas na deflagração e na distribuição dos escorregamentos translacionais rasos na Serra do Mar (SP), alcançou-se aqui resultados relevantes com a indicação de uma possível superfície de ruptura entre $1 \mathrm{~m}$ e 2,5m de profundidade.

Os resultados mostraram que os horizontes de solos mais profundos são formados predominantemente por materiais siltosos e/ou arenosos, enquanto os horizontes superficiais apresentaram-se mais argilosos, ainda que com baixos índices de atividade e por muitas vezes inatividades.

Os valores foram compatíveis com os materiais (solo residual) encontrados na Serra do Mar por outros autores. A variação da granulometria em profundidade e da argila não plástica ou inativa podem influenciar o processo de infiltração no solo e sua instabilidade. Assim como a $\mathrm{K}_{\text {sat }}$ apresentou uma pequena variabilidade, não havendo um padrão para os diferentes perfis. Entretanto, foram identificadas descontinuidades hidráulicas significativas entre 1 e 2,5 m, que coincidiram com aquelas observadas em outros trabalhos na Serra do Mar.

Estudos futuros deverão aprofundar as investigações aqui realizadas, envolvendo, por exemplo, o mapeamento da espessura dos solos e da geologia de detalhe; o aumento no número de pontos ensaiados e, por fim o monitoramento hidrológico dos solos em tempo real.

\section{AGRADECIMENTOS}

Os autores agradecem o apoio financeiro do CNPq (Projeto 480515/2011-5) e da FAPESP (Processo 2014/10109-2). Agradecem os colegas Evandro Daniel e William dos Santos pela ajuda durante os trabalhos de campo. Aos pesquisadores pelas contribuições e discussões: Dra. Maria Cristina Perusi (UNESP), Dr. Nelson F. Fernandes (UFRJ) e Dr. Rodolfo M. Mendes (CEMADEN). Agradecem o Instituto de Geociências da UFRJ pelo empréstimo do Permeâmetro de Guelph, os 
laboratórios de Pedologia da USP e do Instituto Geológico de São Paulo onde foram realizados os ensaios. Finalmente agradecem as críticas e sugestões dos revisores anônimos.

\section{REFERÊNCIAS}

ALMEIDA, F. F. M. Fundamentos Geológicos do Relevo Paulista. Instituto Geográfico e Geológico, São Paulo, pp. 167-263. 1964.

AMARAL JUNIOR, A. F. Mapeamento geotécnico aplicado a análise de processos de movimentos de massa gravitacionais: Costa Verde-RJ - Escala 1:10.000. 2007. p. 191. Vol.1. Dissertação (Mestrado em Engenharia) - Escola de Engenharia de São Carlos, Universidade de São Paulo, São Carlos, 2007.

AVElAR, A. S.; COElHO NETTO, A. N., LACERDA, W. A.; BECKER, L. B.; MENDONÇA, M. B. Mechanisms oh the Recent Catastrophic Landslides in the Mountainous Range of Rio de Janeiro, Brazil. In: The Second World Landslide Forum, 2011, Roma. Anais do The Second World Landslide Forum, p. 1-5. 2011.

BRUGGER, P. J.; EHRLICH, M.; LACERDA, W. A. Movements, piezometric level and rainfall at two natural slopes. In: 2a Conferência Brasileira sobre Estabilidade de Encostas- COBRAE \& 2nd Pan American Symposium on Landslides, v. 1: 13-20. 1997.

CAMPOS, T. M. P.; VARGAS Jr., E. A.; EISENSTEIN, Z. Considerações sobre o processo de instabilização de encostas em solos não-saturados no Rio de Janeiro. In: $1^{\underline{a}}$ Conferência Brasileira sobre Estabilidade de Encostas, Rio de Janeiro: 741-755. 1992.

COLLINS, B.; D. ZNIDARCIC. Triggering Mechanisms of Rainfall Induced Debris Flows In 2a Conferência Brasileira sobre Estabilidade de Encostas/2nd PanAmerican Symposium on Landslides, Rio de Janeiro: 277-286. 1997. 
CRUZ, O. A Serra do Mar e planície na área de Caraguatatuba- contribuição à geomorfologia litorânea tropical. Série Teses e Monografias, 11, IGEOG/USP: 181 p., 1974.

DE PLOEY, Y.; O, CRUZ. Landslides in the Serra do Mar, Brazil. Catena 6: 111 122 p. 1979.

GERSCOVICH, D. M. S.; VARGAS, E. A.; CAMPOS, T. M. P. On the evaluation of unsaturated flow in a natural slope in Rio de Janeiro, Brazil. Engineering Geology, 88: 23-40. 2006.

GRAMANI, M. F. Caracterização Geológico-Geotécnica das Corridas de Detritos ("Debris Flows") no Brasil e Comparação com Alguns Casos Internacionais. 372f. Dissertação (Mestrado em Engenharia Civil). USP, São Paulo, pp. 372. 2001

HASSUI, Y.; MIOTO, J. A.; MORALES, N. Geologia do Pré-Cambriano. In: FALCONI, F. F.; JUNIOR, A. N. (Org.), Solos do Litoral de São Paulo. São Paulo: ABMS, p. 41-67. 1994.

IPT - INSTITUTO DE PESQUISAS TECNOLÓGICAS DO ESTADO DE SÃO PAULO. Indicação preliminar de áreas prioritárias para recomposição da cobertura vegetal na serra do mar na área de Cubatão. IPT, São Paulo. 1986.

IPT - INSTITUTO DE PESQUISAS TECNOLÓGICAS DO ESTADO DE SÃO PAULO. Estudo das instabilizações de encostas da Serra do Mar na região de Cubatão objetivando a caracterização do fenômeno de corrida de lama e da preservação dos seus efeitos. IPT, São Paulo. Relatório 25957, 187p. 1988.

KIEHL, E. J. Manual de edafologia: Relações solo-planta. São Paulo: Ceres, 262p. 1979.

LACERDA, W. A.; EHRLICH, M.; SANTOS JR., O. F. Efeito das variações de poropressão sobre a estabilidade de encostas em solos residuais. In: 2a Conferência Brasileira sobre Estabilidade de Encostas- COBRAE \& 2nd Pan American Symposium on Landslides, 1997, Rio de Janeiro, v. 1: 381-388. 1997. 
MENDES, R. M. Estudo das propriedades geotécnicas de solos residuais não saturados de Ubatuba (SP). Tese (Doutorado em Engenharia), Escola Politécnica, Universidade de São Paulo, 230p. 2008

REYNOLDS, W. D.; ELRICK, D. E. Measurement of field-saturated hydraulic conductivity, sorptivity and the conductivity-pressure head relationship using the "Guelph Permeameter Proceedings, National Water Association Conference on Characterization and Monitoring of Vadose (Unsaturated) Zone. Denver, Colorado- EUA, 25p. 1985.

REYNOLDS, W. D.; ELRICK, D. E.; TOPP, G. C. A reexamination of the constanthead well permeameter method for measuring saturated hydraulic conductivity above the water table. Soil Sci, 136(4):250-268. 1983.

SELBY, M. J. Hillslope: materials \& processes. Oxford: Oxford University Press, 446p. 1993

SIDLE, R. C., PEARCE, A. J.; O'LOUGHLIN, C. L. Hillslope stability and land use. Washington. Water resources monograph, vol. 11, 140 pp. 1985.

SILVA, D. C. O. Aplicação do Modelo Shalstab na Previsão de Deslizamentos em Petrópolis [Rio de Janeiro] 2006. 132f. Dissertação (Mestrado em Engenharia Civil) Universidade Federal do Rio de Janeiro, COPPE. 2006.

TERLIEN, M. T. J. Hydrological landslide triggering in ash-covered slopes of Manizales (Colombia). Geomorphology, 20: 165-175. 1997.

van ASCH, T.; BUMA, J.; VAN BEEK, L. P. H. A view on some hydrological triggering systems in landslides. Geomorphology, 30: 25-32. 1999.

VARGAS, M. Revisão Histórico-Conceitual dos Escorregamentos da Serra do Mar. Solos e Rochas 22: 53-83. 1999.

VIEIRA, B. C.; FERNANDES, N. F. Landslides in Rio de Janeiro: The role played by variations in soil hydraulic conductivity. Hydrological processes, 18: 791-805. 2004. 
PROPRIEDADES FÍSICAS E HIDROLÓGICAS DOS SOLOS E OS ESCORREGAMENTOS RASOS NA SERRA DO MAR PAULISTA

WAKATSUKI T, TANAKA Y; MATSUKURA, Y. Soil slips on weathearing-limited slopes underain by coarse-grained granite or fine-grained gneiss near Seoul, Republic of Korea. Catena 60: 181-203, 2005.

WOLLE, C. M. E C. S. CARVALHO. Taludes Naturais. In F. F. Falconi and A. N. Jr. (eds), Solos do litoral de São Paulo. Associação Brasileira de Mecânica de Solos Núcleo Regional de São Paulo - ABMS, São Paulo, pp. 180-203. 1994.

WOLLE, C. M.; HACHICH, W. Rain-induced landslides in southeastern Brasil. Proceedings of 12th International Conference Soil Mechanics and Foundation Engineering. Rio de Janeiro, v.3: 1639-1642. 1989. 